\title{
Research on the Man-Machine Engineering Theory and the Applications on Furniture Design and Interior Decoration Design
}

\author{
Anmin Liu ${ }^{1}$ \\ ${ }^{1}$ Art Institute of Jiujiang University, \\ JiuJiang,Jiangxi, 332005 China
}

\begin{abstract}
In this paper, we conduct research on the man-machine engineering theory and the applications on furniture design and interior decoration design. Furniture is one of the important media, contacts and relationships between building it by their own different shape and size provide comfortable environment for people. Ergonomics is the study of human activity and the claim size on the surrounding environment, how to make furniture to better serve people's life and work. Our research proposed novel paradigm of the man-machine engineering theory which is meaningful.
\end{abstract}

Keywords: Man-Machine Engineering; Furniture Design; Interior Decoration Design.

\section{Introduction}

Interior space design is the structure of architectural interior space environment for the reasonable design, in order to meet the material and spiritual requirements in our daily life, according to the space environment and the use of planning, the purpose of the use of material technology and aesthetic principle, ergonomics this scale, at the same time also reflects the historical context, environmental air of style and culture connotation, and so on, builds a function reasonable, comfortable and beautiful, to adapt to the user's actions, psychological requirements of the internal environment of space. Furniture is one of the important media, contacts and relationships between building it by their own different shape and size provide comfortable environment for people. Space form is interior space is limited by the physical component space was without form, and we by man-made or natural, given the space of a form or a shape. Space form of space function value is play a decisive role, but also affects the people in the psychological feeling and experience in the space. The shape of indoor environment to intuitive visual and psychological feeling is the first major elements of interior design. Furniture design purpose is to create a tool can meet the demand of people's life and work, therefore in the process of furniture design, how to make the basic scale better adapt to the variation of various positions in the human body in the static became the most important furniture design problem [1].

Ergonomics is the study of human activity and the claim size on the surrounding environment, how to make furniture to better serve people's life and work and how to through the furniture design to meet the people on the health, comfort, and security and so on various aspects of requirements, the application of ergonomics in the furniture design for the safety of the furniture design, comfort, provide strong reference, so it is applied to household to design is very necessary. The man-machine engineering application in furniture design could be roughly separated into the following parts. (1) Because your armrest, tower linear, size and the curvature of the corner of the brain and closely related to human body parts in contact with the seat, so the size of the seat design will directly affect the degree of comfortable seats. Bright type furniture seat upper part of the back of a chair and the lower part and the ratio between the heights of the visitors are very appropriate, maximum practice the ergonomic furniture design of human nature in the idea. (2) For the purpose of the design of 
armrest is in the seat in order to let the human body is in a state of posture, arm can rely on, ensure the stability of posture, the existence of walking design at the same time can also as a pillar of the body got up from a chair. We observed that in the famous furniture officer cap chair, chair and round-backed armchair of roses and other various types of seat design have armrest. Under the ergonomics theory, the height of the armrest should be with the body's natural droop when advisable, high or low will make human body produce the feeling of discomfort. (3) The application of ergonomics in the line architrave design which is more commonly used in bright type furniture is a kind of molds, apply it to drink after meals in the console table, will be higher than desktop edge line architrave, this will prevent vegetable juice or drinks on their clothes, but also can prevent the liquid flow along the table. In indoor space, the use of the material to the human senses such as vision, touch including the shape of the material, color, texture, pattern and severity of cold soft hard attributes which can cause people to different physiological and psychological reaction. In the following figure one, we illustrate the principles general and specific applications of man-machine engineering theory.

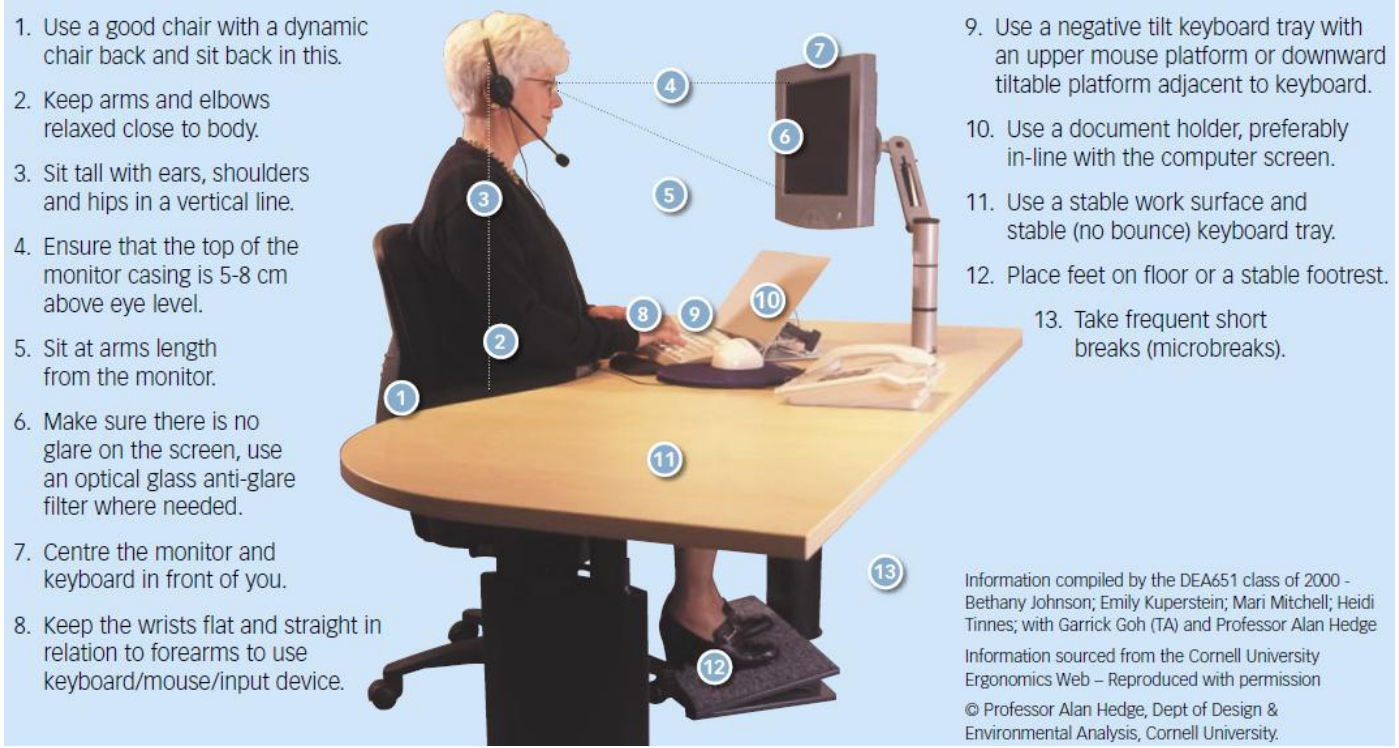

Figure 1. The General and Specific Applications of Man-Machine Engineering Theory

In this paper, we conduct research on the man-machine engineering theory and the applications on furniture design and interior decoration design. Indoor each little space distance size affects People's daily life, a comfortable space size makes people inside activity appears in good order. Such as the width dimension of a corridor if not accord with human body engineering, will appear very crowded, people travel between easy to hit and thus affect the person's psychology in the mood.

\section{Our Proposed Methodology and Approach}

The Principles of the Man-Machine Engineering Theory. "Humanization" design in industrial design must comply with the value of people, serve people need to meet for the purpose of the supremacy. Man's need is various, multi-level basic material needs are met, the spiritual demand of high level becomes the main body, but whether it's physical or mental, industrial products should be in the form of the unity of material and spiritual. Designer to design products to "human nature", make their own design products more color, more 
personality, more likeable, to give the product function designed humanized character, the character, of course, we consumers must rely on itself to feel; Is immeasurable. For the expression of the humanized product design is actually with entity objects to response in the form of invisible spirit. The range of ergonomics research is the design of the product, how to finalize the design of the working environment is more suitable for the people physiological and psychological characteristics which makes people more comfortable and convenient in work, harmony and satisfied. Most industrial products, when the design according to the of all aspects of human physiology and psychological needs to make human nature design, let design combining with people, can better meet the demands of human beings and let people more pleasant. In the rapid development of society, the demands of the human spirit is becoming more and more high, humanized product design to meet the needs of the human spirit, coordination and balance of the human spirit emotion plays a very important role [2].

The Applications on the Interior Decoration Design. Modern indoor environment design is more and more emphasis on people-oriented, emphasis on the relationship between the people and the environment. Therefore, interior design should consider not only the visual environment, but also attaches great importance to the physical environment, physical environment and psychological environment. Now people increasingly attach enough importance to the general environmental color on people's cognitive judgment and the influence of body and mind. The continuation and deepening of architecture design interior designer, architectural design and interior design so it is connected, the style of interior design is often first to a great extent, and architectural design on the aesthetic point of view is consistent, also is very similar on the technique of expression, the integral style of interior design affects the beautiful degree, especially the unity of the style, not the pursuit of diversification, but in the end it is appear desultorily, the style is unified, unified, both inside and outside will be very good improve one space of emotional appeal. In the practice of the decorative design, color design should follow some basic principles, these principles better service make the colors in the design of the space environment, thus creating a harmonious, warm and comfortable color environment.

The core principles could be summarized as the follows. (1) Principle of functional requirements. Because of the different space use function and nature, the size of the space and form, the user's career, sex, hobbies and aesthetic temperament and interest, reflected in the indoor space design of the color also will have a significant difference. In general, the design should consider the following three most important factors to carry on the design. Design should be the first analysis of indoor nature, purpose and function, and then consider the color design according to the specific circumstances and requirements. (2) Harmonious and unified indoor space design of the color is the most important principle. In the interior space, color harmony and contrast is the most basic relationship, harmony and contrast is mutual, both in the interior space is saved, regulate each other be short of one cannot. If indoor only harmony and no contrast change, so the interior space of the color will appear drab and depressing, lack of vigor and vitality [3]. (3) Indoor color and timeliness of the close relationship to the role of a long time in people living or working in the environment, so the indoor color need to consider the feelings of people in it for a long time.

Lighting enables us to see what our environment, it can make us feel good and bad which can make us feel surprised and excited. We can't touch the light, and for a large part of it, and we can't see, we can see the effect of the light, and this is very wonderful. We can use lamplight role in interior design, to strengthen hope to pay 
attention to the places, such as landscape, at the same time can also be used to weaken the attention does not want to be a secondary place, thus further purified to improve and make the space. Decorative lighting is a lighting own light color model as ornamental objects, often using the shot point lights through the colored glass produce all sorts of color formation on the wall. In order to obtain the best visual effect, must consider the following aspects, glare, eyes of aging, adaptive adjustment of light and shade. For adaptive regulating eye to light and shade, we have some experience of life, for example, we have gone from the indoor to dark outside and we may have to spend a few minutes to get the outside scenery see more clearly.

The Applications on Furniture Design. Furniture configuration consists of two parts, and equipped with furniture decorate, in the process of configuration is based on people's behavior activity to determine the function of the space, but also for the realization of the function of space and configuration suitable furniture. The process is on the basis of the size of the bedroom space and space use a line position and people's physiological and psychological characteristics, to scientific and rational layout in indoor furniture suitable bearing and eventually make the person produces intimacy. One of the most important principles is the most direct configuration ergonomics principle. Different categories in indoor environment configuration of furniture itself, can reflect the different design style, color, texture has a great influence on the indoor environment. Sit kind of furniture is one of the most close furniture production and living with people, is the most important part of the furniture design, the design is scientific and reasonable directly affects the people learn, work and rest. Therefore, for modern furniture design, should take human body size as the core, to give full consideration to the human body's size and research of human body size and sit kind of furniture, the relationship between main dimensions and combining with the characteristics of sit kind of furniture design, to meet sit kind of furniture, on the basis of the basic function, make sit kind of furniture design more humanized. Outdoor furniture as the outdoor environment and the design of the product is close to the people [4]. The pros and cons of the design and has a direct relationship. But for a long time, the designer of outdoor furniture in ergonomics considers less application. So, explore how to coordinator, outdoor furniture, and relationship between the environment, achieve the man-machine environment as a whole function optimization. The figure two demonstrates the sample designs.
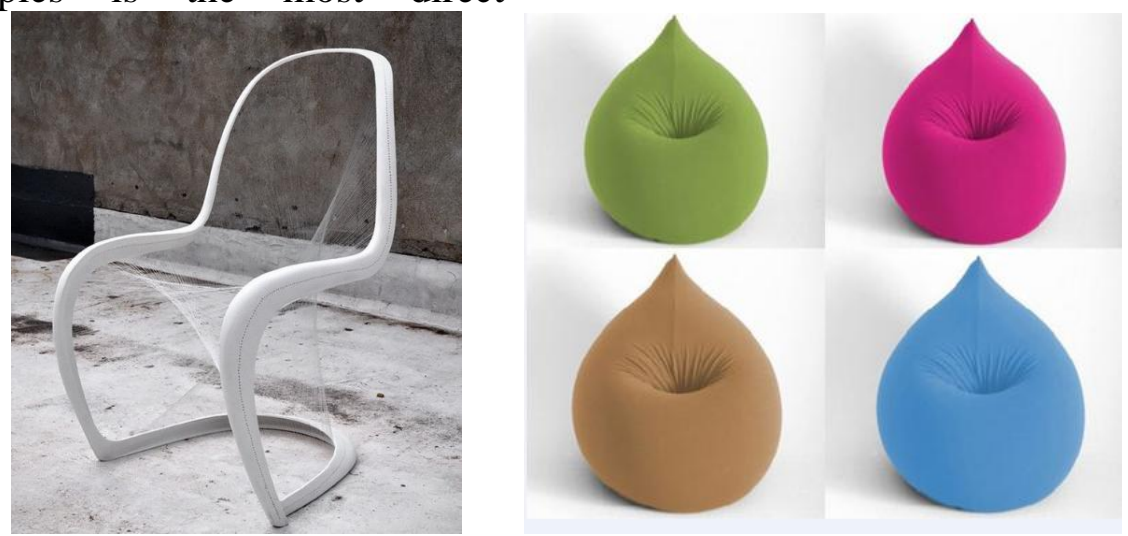

Figure 2. The Demonstration and Illutration of the Applications on Furniture Design

The Prospect and the Development. By using the theory of ergonomics to modern furniture design is inevitable in the development of furniture design. Only fully using the core principle of ergonomics and modern furniture design to make the design of the furniture really become people-oriented design could get the further development. The application of 
ergonomics in the modern furniture design, with particular emphasis on the steps in use process of physiological and psychological reaction of the body, and make scientific experiment and calculation, on the basis of the analysis of a large number of provide scientific basis for furniture design. Comply with the principle of ergonomics humanized design the reality, but also the cutting edge of fashion and trend, is a kind of the embodiment of the humanistic spirit, is the combination of people and modern furniture and perfect harmony, make humanized design truly reflects the respect for people and concern. Therefore, we must strengthen the application of ergonomics principle in modern furniture design research.

\section{Conclusion}

In this paper, we conduct research on the man-machine engineering theory and the applications on furniture design and interior decoration design. Comfort as a kind of subjective feeling, its individual character quite strong. From the philosophical perspective, things no matter how many differences between, there are some connection. In indoor furniture configuration, the application research of comfort is mainly on the spatial relationship, is not confined to one or a certain type of furniture, so the scope of the research is mainly between furniture and furniture, the relationship between furniture and interior space environment. Furniture to reshape the space form, to identify a space, shape space, optimization of space and the space made deeper rich content, show in front of people. The successful interior space should be harmonious environment, furniture and interior space together, complement each other. In the future, we will conduct more research to optimize the current approach.

\section{References}

[1] Sun J, Ren Y. Application of Man-Machine-Environment System Engineering to Create a New World of Changqing Oilfield[J]. Lecture Notes in Electrical Engineering, 2014.

[2] Qin Y T, Cao W Q, Dang-Ke L I, et al. The man-machine-environment reliability evaluation method of cryogenic pneumatic valve[J]. Journal of Rocket Propulsion, 2014.

[3] Hackman J R, Wageman R. A Theory of Team Coaching[J]. Academy of Management Review, 2005, 30(4):41 - 61.

[4] Long S, Dhillon B S. Proceedings of the 13th International Conference on Man-Machine-Environment System Engineering[J]. Lecture Notes in Electrical Engineering,

2014 